\title{
Consistency of self-reported alcohol consumption on randomized and sequential alcohol purchase tasks
}

\author{
Michael Amlung ${ }^{1}$ * and James MacKillop ${ }^{1,2}$ \\ 1 Department of Psychology, The University of Georgia, Athens, GA, USA \\ ${ }^{2}$ Center for Alcohol and Addiction Studies, Brown University, Providence, RI, USA
}

\section{Edited by:}

Paul Verbanck, CHU Brugmann, Belgium

Reviewed by:

Henriette Walter, University of Vienna, Austria

Lorenzo Somaini, Addiction Treatment Centre Local Health Unit Biella, Italy

\section{${ }^{*}$ Correspondence:}

Michael Amlung, Department of Psychology, The University of Georgia, Athens, GA 30602, USA. e-mail: mamlung@uga.edu
Behavioral economic demand for addictive substances is commonly assessed via purchase tasks that measure estimated drug consumption at a range of prices. Purchase tasks typically use escalating prices in sequential order, which may influence performance by providing explicit price reference points. This study investigated the consistency of value preferences on two alcohol purchase tasks that used either a randomized or sequential price order (price range: free to $\$ 30$ per drink) in a sample of 91 young adult monthly drinkers. Randomization of prices significantly reduced relative response consistency $(p<0.01)$, although absolute consistency was high for both versions (>95\%). Self-reported alcohol consumption across prices and indices of demand were highly similar across versions, although a few notable exceptions were found. These results suggest generally high consistency and overlapping performance between randomized and sequential price assessment. Implications for the behavioral economics literature and priorities for future research are discussed.

Keywords: alcohol, purchase task, demand, behavioral economics, consistency

\section{INTRODUCTION}

A behavioral economic approach to substance abuse draws on principles of psychology and economics to characterize how drug use is influenced by a variety of factors, including increases in the cost of obtaining the drug and the presence of alternative reinforcers (Bickel and Vuchinich, 2000; MacKillop et al., 2013). One behavioral economic variable that has received particular focus is substance demand, or the quantitative relationship between drug consumption and its cost (Hursh et al., 2005). In the context of alcohol use disorders (AUDs), demand for alcohol can be efficiently assessed using alcohol purchase tasks (APT; Murphy and MacKillop, 2006), which measure self-reported alcohol consumption at escalating prices. Demand curves generated from an APT reveal that alcohol consumption prototypically decreases as a function of price, with the corresponding expenditure curves conforming to an inverted U-shape. Behavioral economic demand curve analyses can be used to translate the relationship between consumption and cost into multiple indices of motivation, including intensity (i.e., alcohol consumption at minimal cost), breakpoint (i.e., the price that suppresses alcohol consumption to zero), $O_{\max }$ (i.e., the maximum expenditure on alcohol), and $P_{\max }$ (i.e., the price associated with the maximum alcohol expenditure). Finally, quantitative modeling of the demand curve allows for the calculation of elasticity, an index of proportionate price sensitivity (e.g., Hursh and Silberberg, 2008).

As with any new measure, the use of purchase tasks to assess demand for addictive substances requires thorough psychometric validation. Several studies have investigated the concurrent and predictive validity of the motivational indices generated from an APT approach. For example, demand indices are significantly associated with drinking and AUD severity (Murphy and MacKillop,
2006; MacKillop et al., 2010a) and have predicted response to a brief alcohol intervention in young adults (MacKillop and Murphy, 2007). Performance on purchase tasks for alcohol (Murphy et al., 2009) and cigarettes (Few et al., 2012) has also been shown to be temporally stable, exhibiting high test-retest reliability. Furthermore, although purchase tasks typically involve hypothetical outcomes, performance on a hypothetical APT has been shown to correspond with APT performance for actual alcohol and money (Amlung et al., 2012). Finally, performance on purchase tasks has been shown to be influenced by substance-related environmental cues (MacKillop et al., 2010b, in press; Amlung et al., 2012) as well as acute withdrawal states in the case of nicotine dependence (MacKillop et al., in press). Taken together, these studies indicate that these paradigms may provide a meaningful complement to other commonly used measures of drug use motivation.

Given the proliferation of the purchase task approach, understanding how individuals' preferences are influenced by methodological characteristics of the measures themselves is a timely priority for research. For instance, a potential limitation in previous APT studies - and studies using purchase tasks for other commodities - is that demand is typically assessed using an escalating price sequence. Sequential price order provides explicit, item-by-item price reference points, in which choices on previous items may unduly influence responses at the current price. Stated differently, the structured nature of the task itself may artificially inflate response consistency. In this context, consistency refers to the expectation that consumption should decrease as a function of increasing costs (i.e., the law of demand). Randomized assessment, on the other hand, does not readily provide these reference points and may provide a more valid estimate of underlying value preferences. Thus, an important empirical question is the extent 
to which responses on purchase tasks are consistent irrespective of price order.

The goal of the present study was to compare self-reported alcohol consumption across two APT versions, one that used a randomized price sequence and another that used a standard sequential (escalating) price sequence. We hypothesized that, given the absence of clear reference points, participants' consistency of value preferences on the APT would be significantly lower on the randomized version compared to the sequential version. However, we anticipated that overall level of consistency would be generally high across both versions, reflecting clear underlying alcohol-related value preferences.

\section{MATERIALS AND METHODS \\ PARTICIPANTS}

Participants were 91 undergraduates (59\% female; $M$ age $=20.7$, $\mathrm{SD}=2.1 ; 87 \%$ white, $4 \%$ African American, $8 \%$ Asian, $1 \%$ race unreported) who reported drinking alcohol at least monthly. Median annual household income (by participant self-report) was $\$ 97,500$ (inter-quartile range: $\$ 52,500-\$ 120,000)$. Participants drank an average of $12.9(\mathrm{SD}=10.1)$ drinks/week. The data were collected via group assessments that occurred in the evenings in university classrooms. All participants provided informed consent and received research credit or extra credit in psychology courses. All procedures were approved by the University of Georgia Institutional Review Board.

\section{MATERIALS}

Participants completed a comprehensive demographics assessment. Weekly alcohol use was assessed using the Daily Drinking Questionnaire (DDQ; Collins et al., 1985). Demand for alcohol was assessed via a state-based APT procedure based on previous purchase task studies and laboratory self-administration paradigms (see Amlung et al., 2012; MacKillop et al., in press). Participants were asked to estimate how much of a hypothetical $\$ 30$ "bar tab" they would allocate to purchasing their typical alcoholic beverage at 25 prices ( $\$ 0-\$ 30 /$ drink; see Table 1). Consistent with previous research (e.g., Amlung et al., 2012), participants were instructed that each drink was approximately half the size of a standard drink, the maximum number of drinks available was eight, and the total volume would be sufficient to raise their blood alcohol level to $0.07 \%$. They were also told that they would have $1 \mathrm{~h}$ to consume the drinks and that they could not take any leftover drinks with them. These instructions were chosen to maximally standardize consumption parameters and for comparability with other in vivo alcohol self-administration paradigms (O'Malley et al., 2002; Anton et al., 2004).

Participants completed two versions of the APT. Prices were presented in a randomized sequence first (see Table 1), followed by sequential assessment. This order was selected to assess the non-traditional order first and avoid the potential confound of a sequential order priming attributions about the relative value of alcohol. The two APT paradigms were separated by an intervening delay discounting paradigm lasting approximately $20 \mathrm{~min}$ that is described elsewhere (see Amlung and MacKillop, 2011). APT paradigms were presented via a Microsoft PowerPoint ${ }^{\circledR}$ slideshow with programmed transitions. APT choice trials were presented
Table 1 | Comparison of sequential and randomized alcohol purchase tasks.

\begin{tabular}{|c|c|c|c|}
\hline \multicolumn{4}{|c|}{ Self-reported alcohol consumption } \\
\hline Price & Random order & Pearson's $r$ & $t(90)$ \\
\hline Free & 3 & $0.86 * * *$ & n.s. \\
\hline$\$ 0.02$ & 25 & $0.78^{* * *}$ & n.s. \\
\hline$\$ 0.10$ & 11 & $0.74 * * *$ & n.s. \\
\hline$\$ 0.25$ & 7 & $0.86^{* * *}$ & n.s. \\
\hline$\$ 0.50$ & 18 & $0.66^{* * *}$ & n.s. \\
\hline$\$ 0.75$ & 23 & $0.79 * * *$ & n.s. \\
\hline$\$ 1.00$ & 10 & $0.76 * * *$ & n.s. \\
\hline$\$ 2.00$ & 2 & $0.65^{* * *}$ & n.s. \\
\hline$\$ 3.00$ & 16 & $0.87 * * *$ & n.s. \\
\hline$\$ 4.00$ & 14 & $0.85^{* * *}$ & n.s. \\
\hline$\$ 5.00$ & 12 & $0.89 * * *$ & n.s. \\
\hline$\$ 6.00$ & 1 & $0.76 * * *$ & $3.02 * *$ \\
\hline$\$ 7.00$ & 20 & $0.58 * * *$ & n.s. \\
\hline$\$ 8.00$ & 24 & $0.67 * * *$ & n.s. \\
\hline$\$ 9.00$ & 8 & $0.70 * * *$ & $3.14 * *$ \\
\hline$\$ 10.00$ & 17 & $0.58 * * *$ & n.s. \\
\hline$\$ 12.00$ & 13 & $0.63 * * *$ & n.s. \\
\hline$\$ 14.00$ & 21 & $0.74 * * *$ & n.s. \\
\hline$\$ 16.00$ & 5 & $0.50 * * *$ & $2.61 *$ \\
\hline$\$ 18.00$ & 9 & 0.13 & $2.15^{*}$ \\
\hline$\$ 20.00$ & 15 & $0.33^{* *}$ & n.s. \\
\hline$\$ 22.50$ & 22 & $0.70^{* * *}$ & n.s. \\
\hline$\$ 25.00$ & 4 & $0.40 * * *$ & $2.29 *$ \\
\hline$\$ 27.50$ & 6 & $0.57^{* * *}$ & n.s. \\
\hline$\$ 30.00$ & 19 & $0.70 * * *$ & n.s. \\
\hline
\end{tabular}

\begin{tabular}{lll}
\hline \multicolumn{3}{c}{ Demand curve indices } \\
& Pearson's $\boldsymbol{r}$ & $\boldsymbol{t}(\mathbf{9 0})$ \\
\hline Intensity & $0.86^{* * *}$ & n.s. \\
Breakpoint & $0.84^{* * *}$ & n.s. \\
$O_{\max }$ & $0.79^{* * *}$ & $4.42^{* *}$ \\
$P_{\max }$ & $0.66^{* * *}$ & $3.92^{* * *}$ \\
$\alpha$ & $0.84^{* * *}$ & $9.97^{* *}$ \\
\hline
\end{tabular}

$N=91 ;{ }^{*} p<0.05 ;{ }^{* *} p<0.01 ;{ }^{* *} p<0.001$.

for $6 \mathrm{~s}$ per item, followed by a fixation screen lasting $2 \mathrm{~s}$. On each trial, participants were reminded of the maximum number of drinks available for each price. Participants recorded preferred consumption at each price on a paper-based response sheet.

\section{DATA ANALYSIS}

All variables were initially screened for missing data, outliers $(Z s>3.29$ ), and distribution abnormalities (Tabachnick and Fidell, 2001). Elasticity $(\alpha)$ was derived using the non-linear exponential demand curve equation provided by Hursh and Silberberg (2008). Additional facets of demand were generated using an observed values approach (Murphy and MacKillop, 2006), including intensity, breakpoint, $O_{\max }$, and $P_{\max }$. The primary measure of response consistency generated from the APT was the proportion 
of "positive reversals" in consumption. Specifically, this was calculated as the proportion of prices in which consumption increased from a lower price to a higher price (e.g., reflecting inconsistent value preferences). Paired samples $t$-tests and Pearson zero-order correlations were conducted to compare pricewise alcohol consumption, demand indices, and response consistency between the randomized and sequential tasks, with a conventional significance level of $p<0.05$.

\section{RESULTS}

A small number of outliers $(1.2 \%)$ on the demand indices were identified, but were determined to be legitimate responses and recoded as one unit higher than the next lowest non-outlying value (Tabachnick and Fidell, 2001). Demand indices were positively skewed and were transformed using square-root (breakpoint, $\left.O_{\max }, P_{\max }\right)$ or logarithmic $(\alpha)$ transformations. Transformations resulted in non-significant levels of skewness and kurtosis for all demand indices except for intensity, which was not transformed. Across both APT versions, demand for alcohol was prototypic, with consumption decreasing as a function of increasing drink price (Figure 1). Consistent with previous findings (e.g., MacKillop et al., 2010a), the correlations among the demand indices within each of the APT version ranged from moderate to high ( rs $0.30-0.89$, ps $<0.01$ ).
Results of the comparison between APT-Sequential and APTRandom are presented in Table 1. Self-reported alcohol consumption was generally highly congruent across both APT versions, with a few exceptions in which greater consumption was observed on the randomized version at $\$ 6, \$ 9, \$ 16, \$ 18$, and $\$ 25$. Intensity and breakpoint did not significantly differ between versions; however, $O_{\max }, P_{\max }$, and $\alpha$ were significantly higher on the randomized version (see Figure 2). Consumption at each price and demand indices were also generally highly correlated across versions, with the exception of consumption at $\$ 18$.

Participants were generally highly consistent in their selfreported alcohol consumption across both APT versions (overall mean percentage of positive reversals $=2.7 \%, \mathrm{SE}=0.3 \%$ ). The proportion of positive reversals was significantly higher on the APT-Random $(M=5.0 \%, \mathrm{SE}=0.6 \%)$ than the APT-Sequential version $(M=0.4 \%, \mathrm{SE}=0.2 \%)[t(90)=8.6, p<0.001]$, indicating greater inconsistency when prices were randomized.

\section{DISCUSSION}

This study evaluated consistency of self-reported demand for alcohol across two versions of an APT, one that used a randomized order of drink prices and another that used a sequential price order. The results generally support the study's hypotheses. Randomization did significantly reduce response consistency, but, in absolute terms, consumption preferences were highly consistent across both

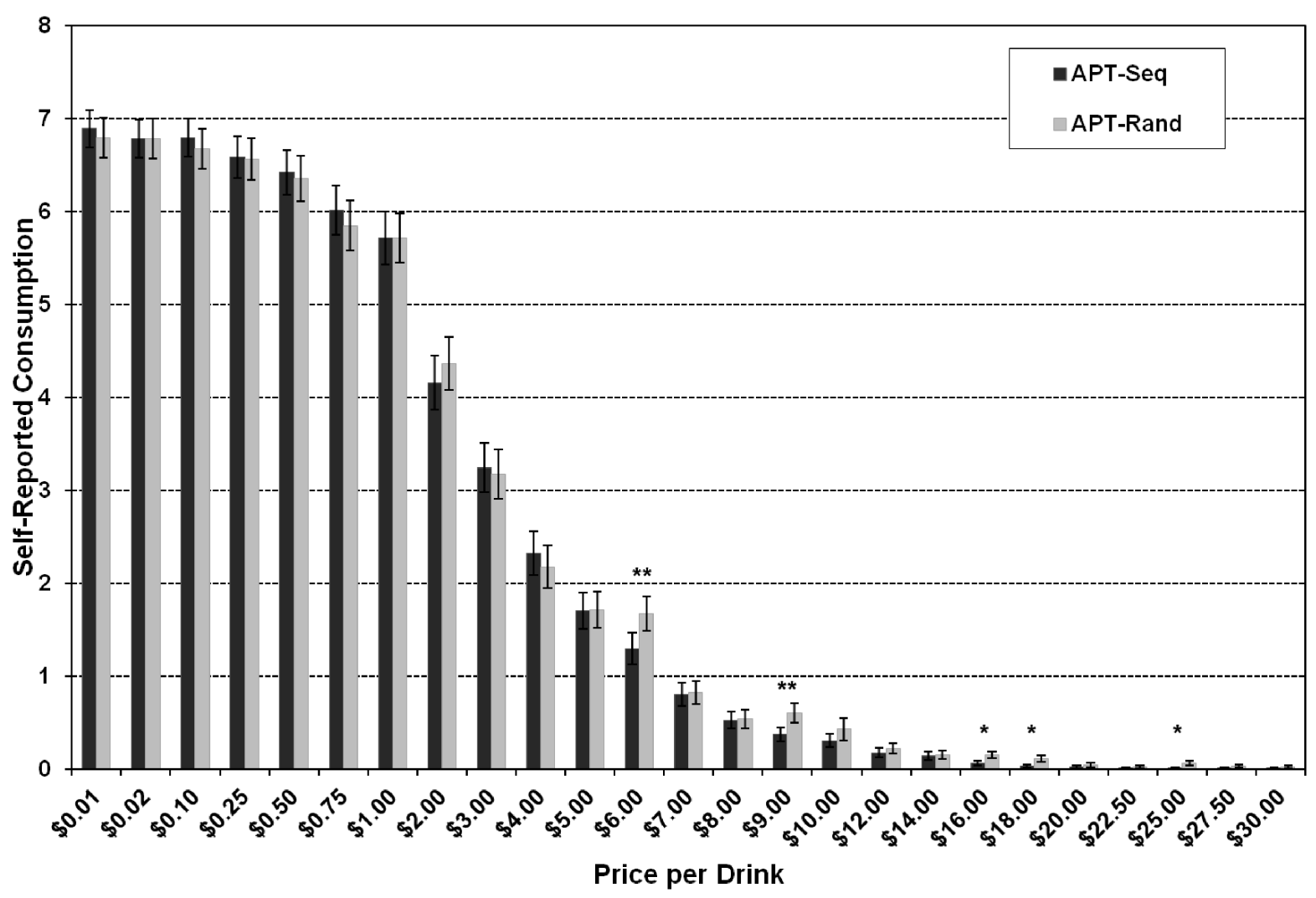

FIGURE 1 | Self-reported alcohol consumption by price across sequential (APT-Seq; dark gray bars) and randomized (APT-Rand; light gray bars) versions. Individual bars represent mean ( \pm standard error). Note, $N=91$; ${ }^{*} p<0.05 ;{ }^{* *} p<0.01$. 

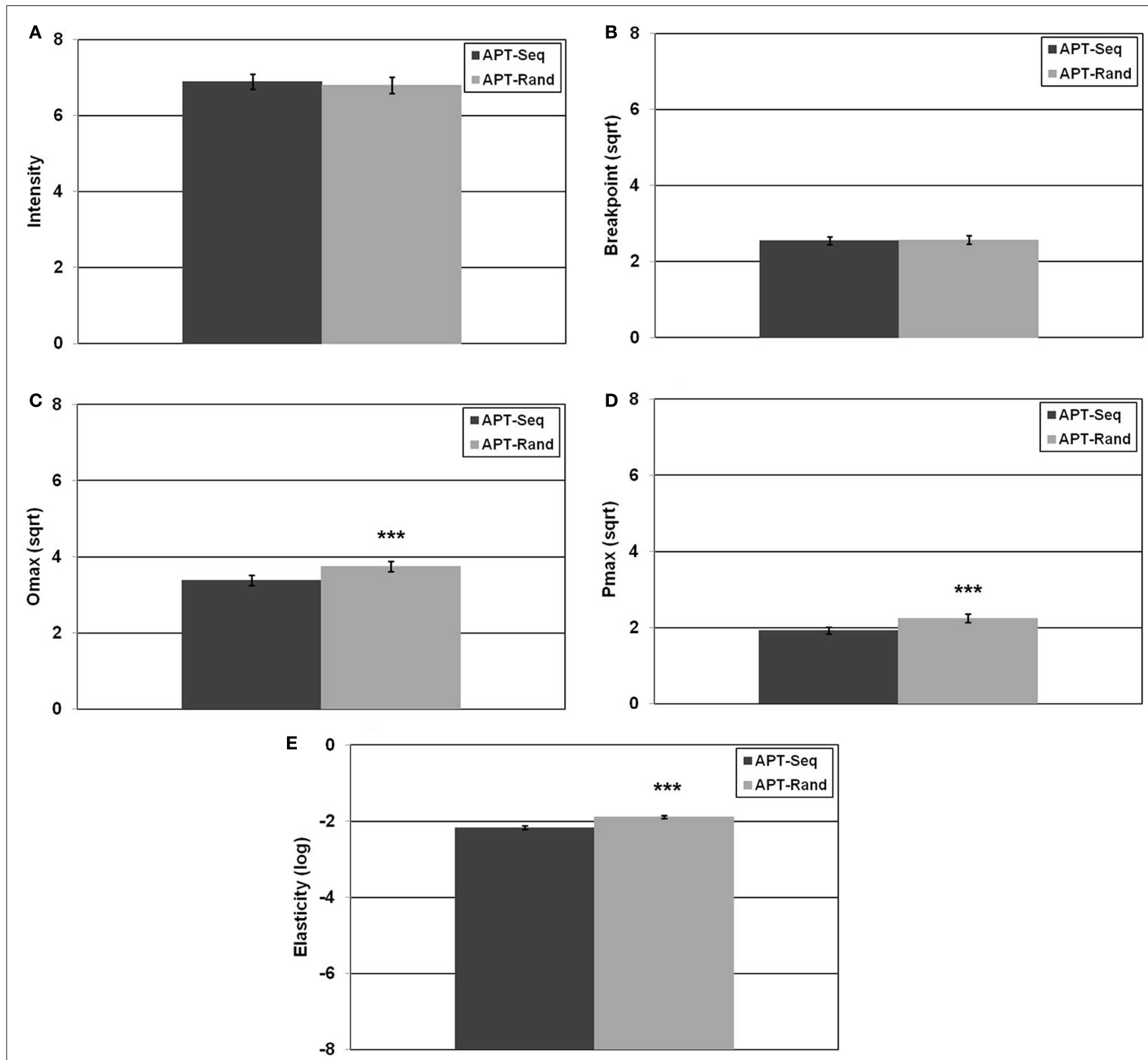

FIGURE 2 | Indices of alcohol demand across sequential (APT-Seq; dark gray bars) and randomized (APT-Rand; light gray bars) versions. (A) Depicts intensity of demand. (B) Depicts square-root transformed breakpoint.
(C) Depicts square-root transformed $O_{\max }$. (D) Depicts square-root transformed $P_{\max }$. (E) Depicts logarithmically transformed elasticity $(\alpha)$. Individual bars represent mean ( \pm standard error). Note, $N=91 ;{ }^{* * *} p<0.001$. versions ( randomized $=95 \%$, sequential $=99 \%$ ). In addition, participants reported highly similar price-level consumption across prices and index-level performance for two of the demand indices (intensity and breakpoint). However, the results also revealed a number of price-level and index-level differences between the two versions. Consumption at several prices and the indices of $O_{\max }$, $P_{\text {max }}$, and elasticity all reflected greater value of alcohol on the randomized version. These findings suggest that sequential order reduces certain indices of the relative value of alcohol, although the magnitude of the effect sizes was relatively small. Taken together, the results suggest generally high consistency and overlapping performance, but differences nonetheless, all reflecting modestly greater value preferences in the randomized version.

With regard to the price-level differences, it is notable that these price intervals were also among the first ones presented (Table 1), particularly in the case of $\$ 6.00$. Higher consumption values on these items may have been because participants were not informed in advance of the range of drink prices prior to completing the assessments. Thus, in the absence of any reference points, performance may be more inconsistent and may reach a "steady state" following exposure to a range of prices. Future studies may improve consistency by explicitly informing participants 
of the range of prices available. Another approach would be to use a pseudo-randomized sequence, with representative prices from low, mid, and high price ranges presented in the first few choices to provide overall reference points.

Interestingly, these findings suggest that participants' consumption behavior at low prices (e.g., intensity) and high prices (e.g., breakpoint) may not be influenced by price order. Indices corresponding to intermediate price intervals of the demand curve (e.g., $O_{\max }$ and $P_{\max }$ ), on the other hand, were significantly augmented by randomized price assessment. Significant differences in elasticity further indicate that participants tended to be more sensitive to changes in cost when prices were arranged randomly. Taken together, these effects support the notion that alcohol preferences at the extremes of the demand curve tend to be most stable, while preferences corresponding to the elastic portion of the curve are more variable and may be affected by price order.

These findings should be interpreted in the context of several considerations. One important consideration of the present study was that the order of APT assessments was not counterbalanced and the time interval between the two APTs was relatively short. An alternative explanation is that the relative value of alcohol simply decreased over time, not as a result of the differing orders. Although this seems to be less likely than the manipulation implemented, future studies should directly address these issues via a fully counterbalanced design. A second potential limitation of this study was the use of a highly structured state-based purchase task paradigm. Although this type of APT paradigm was selected to allow for comparison with previous laboratory-based purchase task studies (Amlung et al., 2012), it is possible that the constraints on the number of drinks available or the instructional set may limit generalization of these findings to trait-based purchase tasks. Investigating the effects of price order in traditional, trait-based purchase tasks would be worthwhile. This study also used hypothetical APTs, though a recent study found close correspondence between hypothetical and actual APT assessments

\section{REFERENCES}

Amlung, M., Acker, J., Stojek, M., Murphy, J. G., and MacKillop, J. (2012). Is talk "cheap"? An initial investigation of the equivalence of alcohol purchase task performance for hypothetical and actual rewards. Alcohol. Clin. Exp. Res. 36, 716-724.

Amlung, M., and MacKillop, J. (2011). Delayed reward discounting and alcohol misuse: the roles of response consistency and reward magnitude. J. Exp. Psychopathology 2, 418-431.

Anton, R. F., Drobes, D. J., Voronin, K., Durazo-Avizu, R., and Moak, D. (2004). Naltrexone effects on alcohol consumption in a clinical laboratory paradigm: temporal effects of drinking. Psychopharmacology (Berl.) 173, 32-40.

Bickel, W. K., and Vuchinich, R. E. (2000). Reframing Health Behavior Change with Behavioral Economics. Associates.

Collins, R. L., Parks, G. A., and Marlatt, G. A. (1985). Social determinants of alcohol consumption: the effects of social interaction and model status on the self-administration of alcohol. J. Consult. Clin. Psychol. 53, 189-200.

Few, L. R., Acker, J. E., Murphy, C. M., and MacKillop, J. (2012). Temporal stability of a cigarette purchase task. Nicotine Tob. Res. 14, 761-765.

Hursh, S. R., Galuska, C. M., Winger, G., and Woods, J. H. (2005). The economics of drug abuse: a quantitative assessment of drug demand. Mol. Interv. 5, 20-28.

Hursh, S. R., and Silberberg, A. (2008). Economic demand and essential value. Psychol. Rev. 115, 186-198.

MacKillop, J., Miranda, R. Jr., Monti, P. M., Ray, L. A., Murphy, J. G.,
Mahwah, NJ: Lawrence Erlbaum

(Amlung et al., 2012). Finally, participants were primarily young adult Caucasian college students, which may constrain the validity of the findings to a high-achieving and possibly more economically minded sample. Future studies should examine effects of APT price order in a larger, more diverse sample, especially in terms of educational status, intellectual functioning, and level of alcohol misuse.

These considerations notwithstanding, this study has potentially important implications for the field of behavioral economics and substance abuse research in general. This study provides the first evidence that randomized APT performance is prototypic and participants' preferences are generally consistent. However, this study also reveals that reference effects may be present in sequential assessment and that individual differences in value are present. Although the existing literature attests to the validity of the sequential version, this study suggests that randomized versions can be used without sacrificing overall response consistency. Laboratory studies investigating demand as a state variable (e.g., MacKillop et al., 2010b, in press; Amlung et al., 2012) might benefit from a reduction in reference-based choices. Future work is needed to replicate and extend the present results in order to maximize the methodological validity of purchase task assessment in addiction research.

\section{ACKNOWLEDGMENTS}

This research was supported by National Institutes of Health grants: K23 AA016936 (James MacKillop) and P30 DA027827 (James MacKillop), and also the University of Georgia Research Foundation (James MacKillop). Generous financial support was also provided by a graduate fellowship by the John and Mary Franklin Foundation (Michael Amlung). The authors wish to thank the graduate students and research assistants of the Experimental and Clinical Psychopharmacology Laboratory in the Department of Psychology at the University of Georgia for their valuable assistance with data collection and analysis.

Rohsenow, D. J., Mcgeary, J. E., Swift, R. M., Tidey, J. W., and Gwaltney, C. J. (2010a). Alcohol demand, delayed reward discounting, and craving in relation to drinking and alcohol use disorders. J. Abnorm. Psychol. 119, 106-114.

MacKillop, J., O'Hagen, S., Lisman, S. A., Murphy, J. G., Ray, L. A., Tidey, J. W., Mcgeary, J. E., and Monti, P. M. (2010b). Behavioral economic analysis of cue-elicited craving for alcohol. Addiction 105, 1599-1607.

MacKillop, J., and Murphy, J. G. (2007). A behavioral economic measure of demand for alcohol predicts brief intervention outcomes. Drug Alcohol Depend. 89, 227-233.

MacKillop, J., Amlung, M., Murphy, C. M., Acker, J., and Ray, L. A. (2013). "A behavioral economic approach to health behavior," in Theory and Practice for a New
Public Health, eds R. Diclemente, L. F. Salazar, and R. A. Crosby (Sudbury, MA: Jones and Bartlett Publishers).

MacKillop, J., Brown, C. L., Stojek, M., Murphy, C. M., Sweet, L., and Niaura, R. (in press). Behavioral economic analysis of withdrawaland cue-elicited craving for tobacco. Nicotine Tob. Res.

Murphy, J. G., and MacKillop, J. (2006). Relative reinforcing efficacy of alcohol among college student drinkers. Exp. Clin. Psychopharmacol. 14, 219-227.

Murphy, J. G., MacKillop, J., Skidmore, J. R., and Pederson, A. A. (2009). Reliability and validity of a demand curve measure of alcohol reinforcement. Exp. Clin. Psychopharmacol. 17, 396-404.

O’Malley, S. S., Krishnan-Sarin, S., Farren, C., Sinha, R., and Kreek, M. J. (2002). Naltrexone decreases craving and alcohol self-administration 
in alcohol-dependent subjects and activates the hypothalamopituitary-adrenocortical axis. Psychopharmacology (Berl.) 160, 19-29.

Tabachnick, B. G., and Fidell, L. S. (2001). Using Multivariate Statistics. Needham Heights, MA: Allyn \& Bacon.
Conflict of Interest Statement: The authors declare that the research was conducted in the absence of any commercial or financial relationships that could be construed as a potential conflict of interest.

Received: 02 April 2012; accepted: 13 June 2012; published online: 02 July 2012.
Citation: Amlung $M$ and MacKillop J (2012) Consistency of selfreported alcohol consumption on randomized and sequential alcohol purchase tasks. Front. Psychiatry 3:65. doi: 10.3389/fpsyt.2012.00065

This article was submitted to Frontiers in Addictive Disorders, a specialty of Frontiers in Psychiatry.
Copyright (c) 2012 Amlung and MacKillop. This is an open-access article distributed under the terms of the Creative Commons Attribution Non Commercial License, which permits non-commercial use, distribution, and reproduction in other forums, provided the original authors and source are credited. 